OPEN ACCESS

Edited by:

Biao Kan,

National Institute for Communicable

Disease Control and Prevention

(China CDC), China

Reviewed by:

Yanpeng Wang,

Chinese Academy of Sciences, China

Alexandra Lianou,

University of Patras, Greece

${ }^{*}$ Correspondence:

Hongning Wang

whongning@163.com

Specialty section:

This article was submitted to

Evolutionary and Genomic Microbiology,

a section of the journal

Frontiers in Microbiology

Received: 24 June 2021

Accepted: 18 August 2021 Published: 16 September 2021

Citation:

Li C, Wang Y, Gao Y, Li C, Ma B and Wang $H$ (2021) Antimicrobial Resistance and CRISPR Typing Among Salmonella Isolates From Poultry Farms in China.

Front. Microbiol. 12:730046. doi: 10.3389/fmicb.2021.730046

\section{Antimicrobial Resistance and CRISPR Typing Among Salmonella Isolates From Poultry Farms in China}

\author{
Cui Li, Yulong Wang, Yufeng Gao, Chao Li, Boheng Ma and Hongning Wang* \\ Animal Disease Prevention and Food Safety Key Laboratory of Sichuan Province, Key Laboratory of Bio-Resource and \\ Eco-Environment of Ministry of Education, College of Life Sciences, Sichuan University, Chengdu, China
}

Although knowledge of the clustered regularly interspaced short palindromic repeat (CRISPR)-Cas system has been applied in many research areas, comprehensive studies of this system in Salmonella, particularly in analysis of antibiotic resistance, have not been reported. In this work, 75 Salmonella isolates obtained from broilers or broilers products were characterized to determine their antimicrobial susceptibilities, antibiotic resistance gene profiles, and CRISPR array diversities, and genotyping was explored. In total, $80.00 \%$ (60/75) of the strains were multidrug resistant, and the main pattern observed in the isolates was CN-AZM-AMP-AMC-CAZ-CIP-ATM-TE-SXT-FOS-C. The resistance genes of streptomycin (aadA), phenicol (floR-like and catB3-like), $\beta$-lactams (bla TEM $_{\text {, bla }}$ OXA, and bla $\left.{ }_{\mathrm{CTX}}\right)$, tetracycline [tet(A)-like], and sulfonamides (sul1 and sul2) appeared at higher frequencies among the corresponding resistant isolates. Subsequently, we analyzed the CRISPR arrays and found 517 unique spacer sequences and 31 unique direct repeat sequences. Based on the CRISPR spacer sequences, we developed a novel typing method, CRISPR locus three spacer sequences typing (CLTSST), to help identify sources of Salmonella outbreaks especially correlated with epidemiological data. Compared with multi-locus sequence typing (MLST), conventional CRISPR typing (CCT), and CRISPR locus spacer pair typing (CLSPT), discrimination using CLTSST was weaker than that using CCT but stronger than that using MLST and CLSPT. In addition, we also found that there were no close correlations between CRISPR loci and antibiotics but had close correlations between CRISPR loci and antibiotic resistance genes in Salmonella isolates.

Keywords: Salmonella, CRISPR-Cas, antimicrobial resistance, molecular typing, CRISPR array

\section{INTRODUCTION}

Salmonella is one of the main causes of bacterial gastroenteritis worldwide. To date, more than 2,600 Salmonella serotypes have been reported globally (Graziani et al., 2017). Moreover, approximately one-tenth of individuals worldwide are infected by non-typhoidal Salmonella enterica isolates annually resulting in 33 million deaths [Salmonella (non-typhoidal), 2018]. However, among Salmonella serotypes, only a limited number causes human infection (Uzzau et al., 2000), such as Salmonella enterica serotype Enteritidis (S. Enteritidis) and Salmonella enterica serotype Typhimurium (S. Typhimurium); identification of these infective serotypes is important for public health. Salmonella 
is widespread in domestic and wild animals, particularly poultry and poultry products which have been identified as important sources of human salmonellosis (Sanchez et al., 2002; Antunes et al., 2016; McWhorter and Chousalkar, 2019). For example, in 2018, approximately 207 million eggs from Rose Acre Farms (Seymour, Indiana, United States) were contaminated with Salmonella enterica serotype Braenderup, infecting 45 people, and were recalled across the United States (Centers for Disease Control and Prevention, 2018a). Similarly, eggs from Gravel Ridge Farms (Cullman, Alabama, United States) contaminated with S. Enteritidis, infected 44 people and had to be recalled (Centers for Disease Control and Prevention, 2018b), and a raw frozen chicken product contaminated with multidrug-resistant Salmonella enterica serotype Infantis (S. Infantis) infected 129 people (Centers for Disease Control and Prevention, 2019). Currently, the most commonly used treatment method for Salmonella infection is antibiotic therapy. However, the unnecessary long-term use of antibiotics has led to the development of Salmonella resistance, which poses a major challenge to the treatment of salmonellosis. In particular, there are many serotypes of Salmonella, how to quickly type the strain and trace the source of salmonellosis outbreaks remains to be resolved.

Different molecular typing techniques, such as pulse-field gel electrophoresis (PFGE), multi-locus sequence typing (MLST), and multi-locus variable number tandem repeat (VNTR) analysis, have been developed to track the origins of bacterial disease. PFGE typing has a high resolution for analyzing the homology of pathogenic bacteria from different sources, tracing the origin of foodborne disease outbreaks, determining the diversity of pathogenic bacteria in food, and evaluating the relationships between pathogenic bacteria in food and the related environment (Pang et al., 2007; Best et al., 2009). However, there are no uniform standards for naming, making it difficult to share data and to distinguish among the long-term evolution process from small changes between different strains of the same clone. Although MLST technology compensates for the deficiency of PFGE, it has some limitations for the application of typing in some highly homologous pathogens, due to the conservation of housekeeping genes (Turki et al., 2014; Campioni et al., 2015).

In recent years, innovative and powerful typing methods based on clustered regularly interspaced short palindromic repeat (CRISPR) loci have been developed (Barrangou and Dudley, 2016) and applied to the typing of a variety of bacteria, such as Salmonella (Fabre et al., 2012) and Escherichia coli (Long et al., 2020). Through the study of S. Heidelberg typing, it is found that CRISPR typing can be useful for Salmonella foodborne outbreaks subtyping as well as serve as a complimentary tool to determine source attribution in foodborne outbreaks (Vincent et al., 2018; Yousfi et al., 2020). Researchers combined the multi-virulence gene locus sequence typing (MVLST) scheme with CRISPR typing to develop a CRISPRMVLST method, which was used to genotype 171 Salmonella strains from nine serotypes. The results of this method were better than CRISPR and MLST and could be used as an important genotyping method in Salmonella outbreaks (Liu et al., 2011). Upon comparing the effects of PFGE and CRISPR-MVLST on typing 84 strains of Salmonella enterica serotype Newport, researchers found that the Simpson index D values of the two methods were both higher than 0.95 , suggesting complementary with each other in practice (Shariat et al., 2013b). A new typing method, CRISPR locus spacer pair typing (CLSPT), analyzes only the two newly incorporated spacers adjoining the leader sequence in the two CRISPR loci. Use of this method to analyze 82 Salmonella strains of 21 serotypes showed that although this method was weaker than conventional CRISPR typing (CCT) and PFGE typing, it was better than MLST and did not require analysis of the full CRISPR sequence map; it was also low cost and more practical, requiring only simple operation procedures (Li et al., 2014). Sequencing of 156 Salmonella strains showed that the evolutionary pattern reflected by the CRISPR locus was different from that of phylogeny, and horizontal gene transfer and changes in the shared environment could affect the phylogenetic distribution of Salmonella (Timme et al., 2013). Among these above methods, it is either cumbersome or ignoring the differences in spacer sequences. Thus in order to make up for this shortcoming, a novel method should be developed.

Since the discovery of the CRISPR locus in the 1980s, the functions and applications of CRISPR in prokaryotes have attracted much attention. For example, its spacer sequence has been used as an auxiliary tool for a variety of bacterial typing studies, epidemiological investigations, and evolutionary analyses. The CRISPR array has a high degree of polymorphism, which includes the unique biological and geographical characteristics of bacteria. However, few studies have reported its relationship with bacterial drug resistance, and the results of these studies in different bacteria have been inconsistent. For example, in Klebsiella pneumonia, Neisseria meningitidis, and Vibrio cholera, partly antibiotic resistance genes (ARGs) were positively associated with CRISPR-Cas system (Shehreen et al., 2019). But it is interesting that on the time scale of evolution, CRISPR-Cas has no significant effect on horizontal gene transfers (Gophna et al., 2015). It also has proved that the CRISPR-Cas system in E. coli does not affect the spread of plasmid and antibiotic (Touchon et al., 2012). And Shigella strains could regulate the activity of the CRISPR-Cas system by the insertion sequence (IS) elements that were identified in cas genes and then acquired exogenous resistance genes (Chen et al., 2019). In Salmonella, genome analysis has suggested that generally there are two CRISPR loci and a single type of CRISPR-Cas system, type I (mainly I-E) (Jansen et al., 2002; Grissa et al., 2007). Therefore, in this study, we aimed to analyze the occurrence and diversity of CRISPR arrays using whole-genome sequencing (WGS) data from 75 Salmonella isolates. Additionally, we established a new typing method based on CRISPR for Salmonella, and we further explored the relationships between the CRISPR-Cas system and drug resistance in Salmonella.

\section{MATERIALS AND METHODS}

\section{Salmonella Strains and Antimicrobial Susceptibility Testing}

In total, 75 Salmonella isolates were collected from different broilers or broilers products in eight poultry farms at three provinces (Hebei, Sichuan, and Shandong) and Chongqing municipality of China between 2018 and 2019. Salmonella 
isolation was performed as previously reported (Cai et al., 2016). In brief, first, the pre-enrichment step of Salmonella was carried out by culturing the samples in $10 \mathrm{~mL}$ buffered peptone water (BPW, Beijing Land Bridge Technology Co., Ltd., China) at $37{ }^{\circ} \mathrm{C}$ with $180 \mathrm{rpm}$ for $16 \mathrm{~h}$. Then, $100 \mu \mathrm{L}$ of the BPW was cultured in $10 \mathrm{~mL}$ Rappaport's Broth (MM, Bejing Land Bridge Technology Co., Ltd., China) at $42{ }^{\circ} \mathrm{C}$ with $180 \mathrm{rpm}$ for $24 \mathrm{~h}$. Subsequently, the MM culture was streaked into xylose lysine tergitol 4 (XLT4, Bejing Land Bridge Technology Co., Ltd., China) at $37^{\circ} \mathrm{C}$ for $36 \mathrm{~h}$ in a microbial incubator.

Immunological serotyping of Salmonella isolates was confirmed by slide agglutination for flagellar $(\mathrm{H})$ and somatic $(\mathrm{O})$ antigens using commercially available antisera (Tianrun Bio-Pharmaceutical, Ningbo, China), according to the manufacturer's instructions. The antibiotic susceptibility of these isolates was tested using the disk diffusion method on Mueller-Hinton agar (MHA) according to the guidelines provided by the Clinical and Laboratory Standards Institute (CLSI) (Clinical and Laboratory Standards Institute, 2019), including gentamycin $(\mathrm{CN}, 10 \mu \mathrm{g})$, azithromycin (AZM, 15 $\mu \mathrm{g}$ ), ampicillin (AMP, $10 \mu \mathrm{g}$ ), amoxicillin/clavulanic acid (AMC, 20/10 $\mu \mathrm{g}$ ), ampicillin/sulbactam (SAM, 10/10 $\mu \mathrm{g}$ ), cefoxitin (FOX, 30 $\mu \mathrm{g}$ ), ceftazidime (CAZ, $30 \mu \mathrm{g})$, polymyxin B (PB, 300 IU), meropenem (MEM, $10 \mu \mathrm{g})$, ceftazidime (CIP, $5 \mu \mathrm{g}$ ), aztreonam (ATM, 30 $\mu \mathrm{g}$ ), tetracycline (TE, 30 $\mathrm{g}$ ), trimethoprim/sulfamethoxazole (SXT, $1.25 / 23.75 \mu \mathrm{g}$ ), chloramphenicol (C, 30 $\mu \mathrm{g}$ ), fosfomycin (FOS, $200 \mu \mathrm{g}$ ),

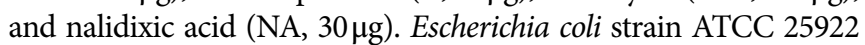
was used as a quality control organism in all antimicrobial susceptibility tests.

\section{WGS and Analysis of Antibiotic Resistance Genes}

Genomic DNA was extracted from Salmonella isolates using a TIANamp Bacteria DNA Kit (Tiangen, Beijing, China), and WGS was carried out by Novogene Co., Ltd., Beijing, China. The sequencing results were analyzed on an Illumina MiSeq platform using 150 base paired-end reads, according to the manufacturer's instructions. The gene fragments were assembled using SPAdes 3.13.0 (Bankevich et al., 2012). Next, the 75 Salmonella genome sequences were submitted to the Center for Genomic Epidemiology (CGE) ${ }^{1}$ and analyzed for ARGs.

\section{Analysis of CRISPR Arrays and Cas Genes}

All CRISPR sequences were analyzed with CRISPRCasFinder ${ }^{2}$, which could be used to identify CRISPR arrays, cas genes, and the CRISPR-Cas type and subtype (Couvin et al., 2018). The results were visualized using CRISPRviz (Nethery and Barrangou, 2019). Weblogo ${ }^{3}$ was used for the analysis of the conservation of leader sequences and direct repeat (DR) sequences (Crooks et al., 2004). Direct repeat sequences were classified according to v1.3.0-2013 in the CRISPRmap (Lange et al., 2013). Secondary structure and minimum free energy (MFE) predictions were

${ }^{1}$ https://cge.cbs.dtu.dk/services/ResFinder/

${ }^{2} \mathrm{https} / / /$ crisprcas.i2bc.paris-saclay.fr/CrisprCasFinder/Index

${ }^{3}$ http://weblogo.berkeley.edu/logo.cgi performed using the RNAfold web server ${ }^{4}$ (Mathews et al., 2004). CRISPRTarget $^{5}$ was used to analyze spacer sequences (Biswas et al., 2013) and the cutoff score was 20. An unweighted pair group method with arithmetic mean (UPGMA) phylogenetic tree based on the CRISPR sequences was constructed to depict the clustering of subtypes determined by CRISPR diversity.

\section{Multi-Locus Sequence Typing (MLST)}

The typing scheme utilized conserved sequences within the housekeeping genes (aroC, dnaN, hemD, hisD, purE, sucA, and thrA). The 75 Salmonella genome sequences were submitted to CGE, and MLST analyses were carried out on the CGE using MLST $2.0^{6}$. The minimum spanning tree was constructed using Phyloviz with the goeBURST Full MST algorithm based on the seven MLST loci. The discrimination index was evaluated based on Simpson's index of diversity using a previously described equation (Hunter and Gaston, 1988).

\section{Conventional CRISPR Typing (CCT)}

First, all spacer sequences in the two gene sequences of CRISPR1 and CRISPR2 were extracted, and the CRISPR1 and CRISPR2 spacer sequence profiles were then drawn and visualized using CRISPRviz.

\section{CRISPR Locus Spacer Pair Typing (CLSPT)}

A spacer sequence in CRISPR1 and CRISPR2 was used, and this spacer sequence was the first spacer sequence close to the leader sequence. The first spacer sequence of the CRISPR1 leader sequence was combined with the first spacer sequence of the CRISPR2 leader sequence. These two spacer sequences were used as the total sequence for Salmonella strain typing.

\section{CRISPR Locus Three Spacer Sequences Typing (CLTSST)}

During the evolution of the strain, the first captured exogenous nucleotide sequence displayed the original information for the strain, and the spacers of the same serotype had a certain degree of conservation. Therefore, this feature could be used to distinguish different serotype clusters. Thus, we developed a new method for Salmonella typing, called as CLTSST. Three spacer sequences that were the initial two spacer sequences (the furthest distance to the leader sequence) and latest spacer sequence close to the leader sequence were extracted, and these spacer sequences were then combined as the total sequence for strain typing.

\section{RESULTS}

\section{Serotyping and Antibiotic Susceptibility of Salmonella}

Thirteen Salmonella enterica serotypes were identified from the 75 Salmonella isolates, including four strains of $S$. Enteritidis,

${ }^{4}$ http://rna.tbi.univie.ac.at/cgi-bin/RNAfold.cgi

${ }^{5}$ http://bioanalysis.otago.ac.nz/CRISPRTarget/crispr_analysis.html

${ }^{6} \mathrm{https://cge.cbs.dtu.dk/services/MLST/}$ 
TABLE 1 | Antibiotic-resistant profiles of Salmonella isolates to different antibiotics.

Antibiotic-resistant profiles ${ }^{a}$

CN-AZM-AMP-AMC-CAZ-CIP-ATM-TE-SXT-FOS-C

CN-AZM-AMP-AMC-CAZ-CIP-ATM-SXT-C-NA

CN-AZM-AMP-AMC-CAZ-ATM-TE-SXT-FOS-C

CN-AMP-AMC-CAZ-CIP-ATM-TE-SXT-FOS-C

AMP-AMC-CAZ-CIP-ATM-TE-SXT-FOS-C

CN-AMP-AMC-PB-CIP-TE-SXT-FOS-C-NA

CN-AMP-AMC-CAZ-CIP-ATM-SXT-FOS-C

CN-AZM-AMP-CAZ-CIP-TE-SXT-C

CN-AMP-AMC-CIP-TE-SXT-C-NA

CN-AMP-AMC-CIP-SXT-C-NA

AMP-AMC-CIP-TE-SXT-C

CN-AMP-CAZ-TE-SXT-C

CN-AMP-AMC-CIP-TE-C

AMP-CAZ-CIP-TE-C-NA

AMP-CIP-TE-SXT-C-NA

AMP-CAZ-ATM-FOS-C

AMP-CIP-SXT-C-NA

AMP-CIP-TE-SXT-C

CIP-TE-SXT-C

AMP-TE-C

TE-SXT-C

AMP-TE

FOS

AMP

none

${ }^{a} C N$, gentamycin; AZM, azithromycin; AMP, ampicillin; AMC, amoxicillin/clavulanic acid; SAM, ampicillin/sulbactam; FOX, cefoxitin; CAZ, ceftazidime; $P B$, polymyxin B; MEM, meropenem; CIP, ciprofloxacin; ATM, aztreonam; TE, tetracycline; SXT, trimethoprim/ sulfamethoxazole; C, chloramphenicol; FOS, fosfomycin, and NA, nalidixic acid.

20 strains of $S$. Indiana, 11 strains of S. Derby, six strains of S. Senftenberg, three strains of S. Kentucky, 24 strains of S. Typhimurium, and other common epidemic serotypes (Supplementary Table S1).

The antimicrobial susceptibilities of 75 Salmonella isolates were observed (Table 1). More than two-thirds (69.33-80.00\%) of the isolates were resistant to AMP, SXT, C, TE, and CIP, whereas almost half (40.00-42.67\%) of the isolates were resistant to $\mathrm{CN}, \mathrm{AMC}, \mathrm{FOS}$, and CAZ. Nearly one-third (36.00\%) of the tested isolates exhibited resistance to ATM. Approximately one-quarter (22.67-25.33\%) of the isolates was resistant to NA and AZM. The resistance rates to PB, MEM, SAM, and FOX were relatively low $(0.00-2.67 \%)$. The drug resistance spectrum of the isolated bacteria (Table 1) showed that $80.00 \%(60 / 75)$ of the strains were multidrug-resistant bacteria that were resistant to three or more drug types, reflecting that antibiotic resistance in the isolated bacteria was prevalent with 25 different resistance patterns identified (Table 1). The main pattern in 14 of the isolates was CN-AZM-AMP-AMC-CAZ-CIP-ATM-TE-SXTFOS-C. However, serotypes and resistance patterns were not correlated, and 47 resistance-associated genes were found in all isolates (Figure 1 and Supplementary Table S2). Among these resistance genes, sul1, sul2, tet(A)-like, bla $a_{\mathrm{TEM}}$, bla $a_{\mathrm{CTX}}$, $b l a_{\mathrm{OXA}}$, floR-like, catB3-like, aac(3)-Iva-like, aph(4)-Ia, and fosA were found in Salmonella isolates at rates of 60.00, 62.67, 45.33, $44.00,40.00,41.33,52.00,40.00,40.00,40.00$, and $37.33 \%$, respectively.

\section{MLST Analysis}

Multi-locus sequence typing classification showed that the 75 Salmonella isolates belonged to 15 ST types (Supplementary Table S1): ST17 (20/75, 26.67\%); ST34 (15/75, $20.00 \%) ;$ ST40 (11/75, 14.67\%); ST19 (9/75, 12.00\%); ST14 $(6 / 75,8.00 \%)$; ST92, ST11, ST96, ST543, and ST2709 (2/75, 2.67\%); ST32, ST413, ST22, ST13, and ST198 (1/75, 1.33\%). The STs of S. Indiana and S. Senftenberg were consistent with their serotypes. The $S$. Typhimurium isolates were divided into six STs, the S. Enteritidis isolates were divided into two STs, and $S$. Kentucky and S. Derby isolates were divided into three STs. The results of the minimum spanning tree-based MLST showed that ST34 was a cloned progenitor and that ST40 evolved from the central ST34 lineage (Figure 2). ST11 isolates were subcloned from the isolate ST40. ST22, ST413, ST198, and ST92 had close genetic relationships, whereas the other STs had close genetic relationships with ST34.

\section{Analysis of the CRISPR-Cas System in Salmonella}

Among the 75 Salmonella isolates, 73 strains had the same general type I-E structure (mostly CRISPR1-Cas system), comprising CRISPR array, leader sequence and cas genes (cas2, cas1, cas6, cas5, cas7, cse2, cse1, and cas3). One of the other two had only two orphan CRISPR arrays and the other had no CRISPR-Cas system. The upstream 5' end of the CRISPR array was adjacent to a leader sequence, which could act as a promoter for the pre-crRNA synthesis. Alignment of the leader sequence of approximately $100 \mathrm{bp}$ adjacent to the upstream CRISPR array showed that there were large differences in CRISPR1 leaders and that the CRISPR2 leaders were more conserved than the CRISPR1 leaders (Figures 3A, B). The DR sequences of CRISPR1 and CRISPR2 were similar. In the sequence comparison and conservation analysis of DR sequences among all CRISPR arrays (evidence level 3 or 4 in CRISPRCasFinder), 31 types of relatively conserved DR sequences were found, ranging in length from 24 to $29 \mathrm{bp}$, with most being 29bp (Figure 3C and Supplementary Table S1). By analyzing these 31 types of DR sequences using CRISPRmap, we found that five DRs had motifs, including four motifs 1 and one motif 2 , and 26 could be classified as existing families, including 21 families 2 and five family 4, suggesting that these DRs were involved in gene expression or DNA metabolism. The results of the MFE secondary structure and MFE prediction showed that a stable stem loop structure was formed by all 31 types of DRs (Figures 3D-F).

In the CRISPR array, the highest numbers of spacers were 33 in CRISPR1 and 34 in CRISPR2, whereas the lowest numbers were seven in CRISPR1 and four in CRISPR2, for a total of 1,607 spacer sequences in CRISPR1 and 1,226 in CRISPR2. After removing duplicates, 517 unique sequences were obtained (274 in CRISPR1 and 243 in CRISPR2), and the length of sequences ranged from 29 to $70 \mathrm{bp}$, with the majority being $32 \mathrm{bp}$. Target prediction was performed on the above 517 spacer sequences using CRISPRTarget. Of these, sixty-one (11.79\%) were found to be homologous to bacteriophages or viruses, and 137 (26.49\%) were homologous to plasmids. Other spacers 


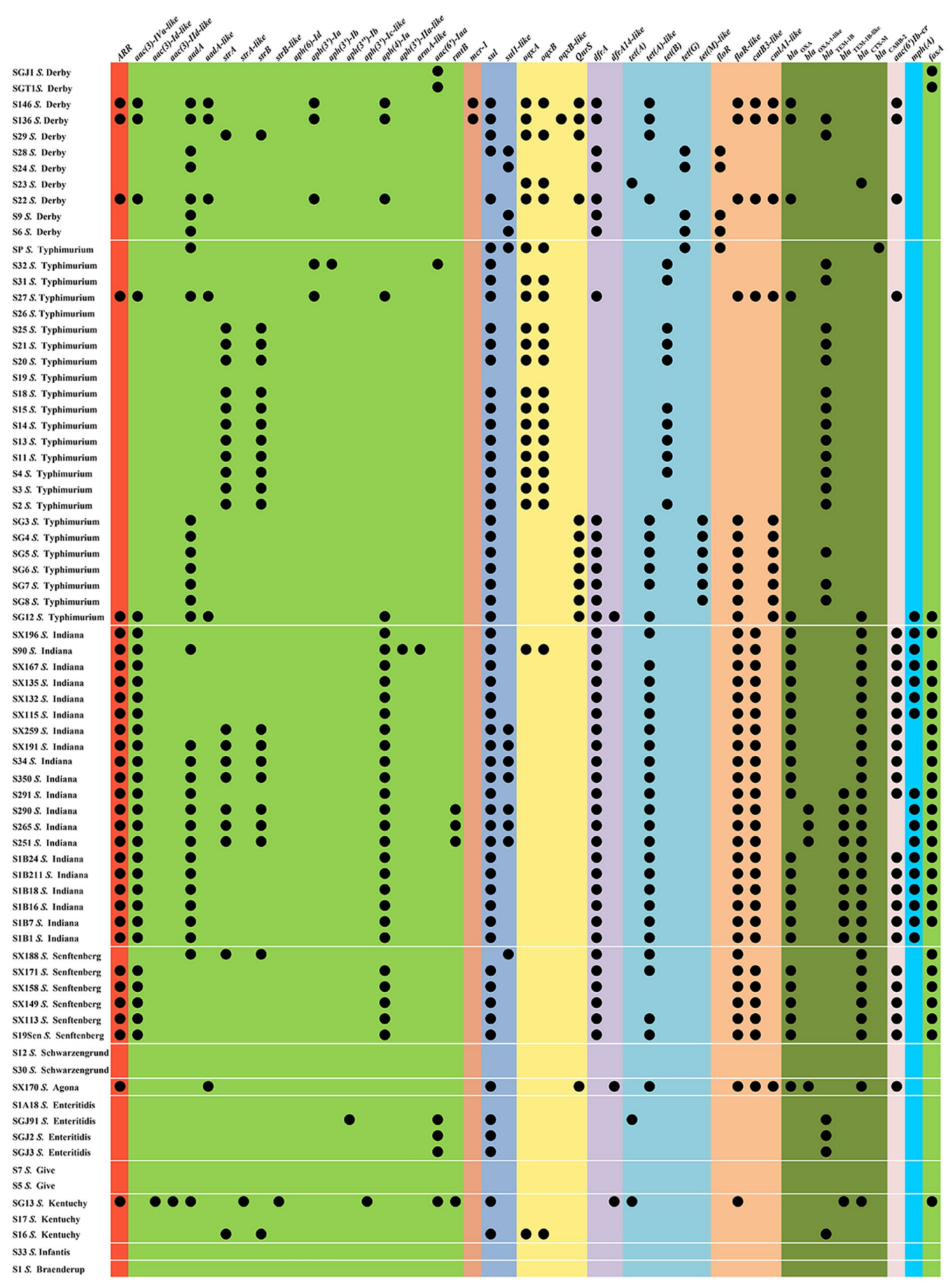

FIGURE 1 | Resistance gene profiles from the Salmonella isolates.

targeted bacteria genes of the non-CRISPR array portions of their own genome, which covered all aspects of bacterial life activities, such as genetic information storage and processing, bacterial functional activities, and metabolism. Many genes associated with antimicrobial function, such as multidrug excretion proteins, and penicillin-binding proteins were also found to be homologous.

\section{CRISPR Typing Analysis}

CRISPR typing was performed by combining CRISPR1 and CRISPR2. The resulting profile for each strain after the spacer sequence was arrayed in CCT, as shown in Figure 4, and the strains were found to have high genetic polymorphism. The arrangement of CRISPR spacers of the same serotype was similar or identical. In total, the 72 Salmonella strains that had two CRISPR loci were divided 


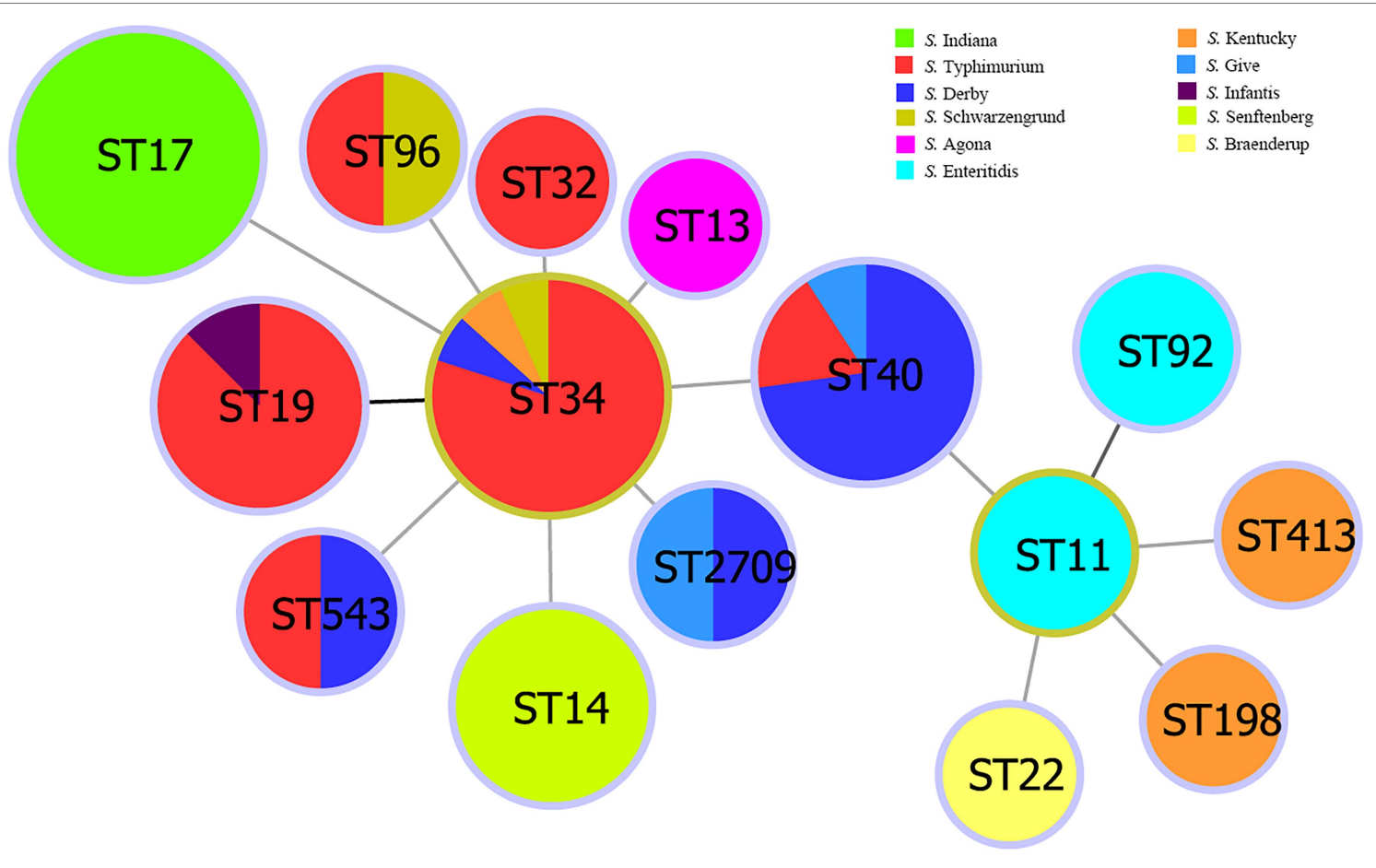

FIGURE 2 | Minimum spanning tree of Salmonella isolates from multi-locus sequence typing data.

A

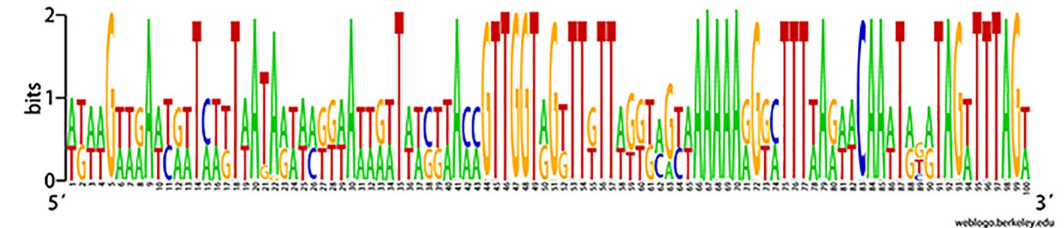

B

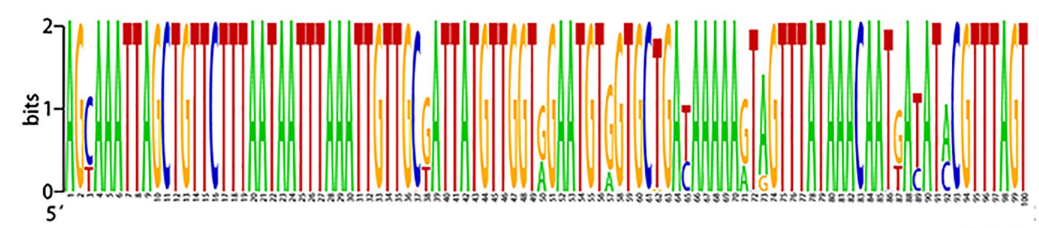

C

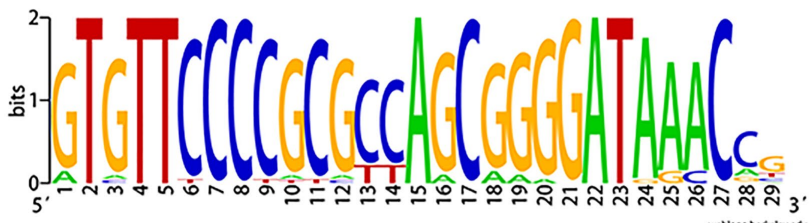

D

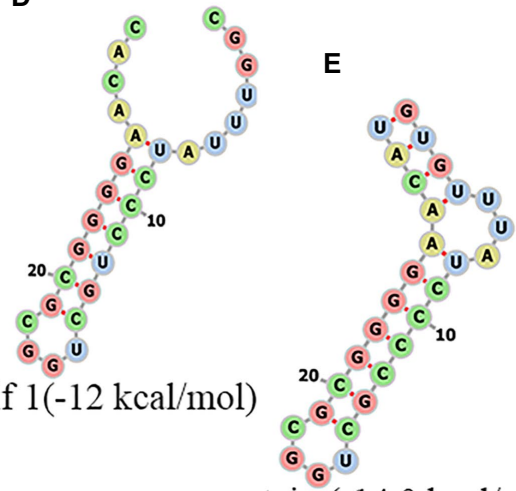

$\mathbf{F}$ uncertain $(-14.9 \mathrm{kcal} / \mathrm{mol})$

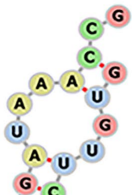

20. G. C

G. $c$

c. $\operatorname{cc}$ motif $2(-15.2 \mathrm{kcal} / \mathrm{mol})$

A. C. 10

c. 6

FIGURE 3 | Analysis of clustered regularly interspaced short palindromic repeat (CRISPR) leader and direct sequences (DRs). (A,B) Conservation analysis of leader sequences of CRISPR1 and CRISPR2, respectively. (C) Conservation analysis of DR sequences. Each logo is consisted of stacks of letters, one stack for each position in the sequence. The height of letters within each stack is measured in bits, with a maximum of two, and reflects the corresponding nucleotide conservation at that position. (D-F) Predicted secondary structure and MEF of a partial DR. 


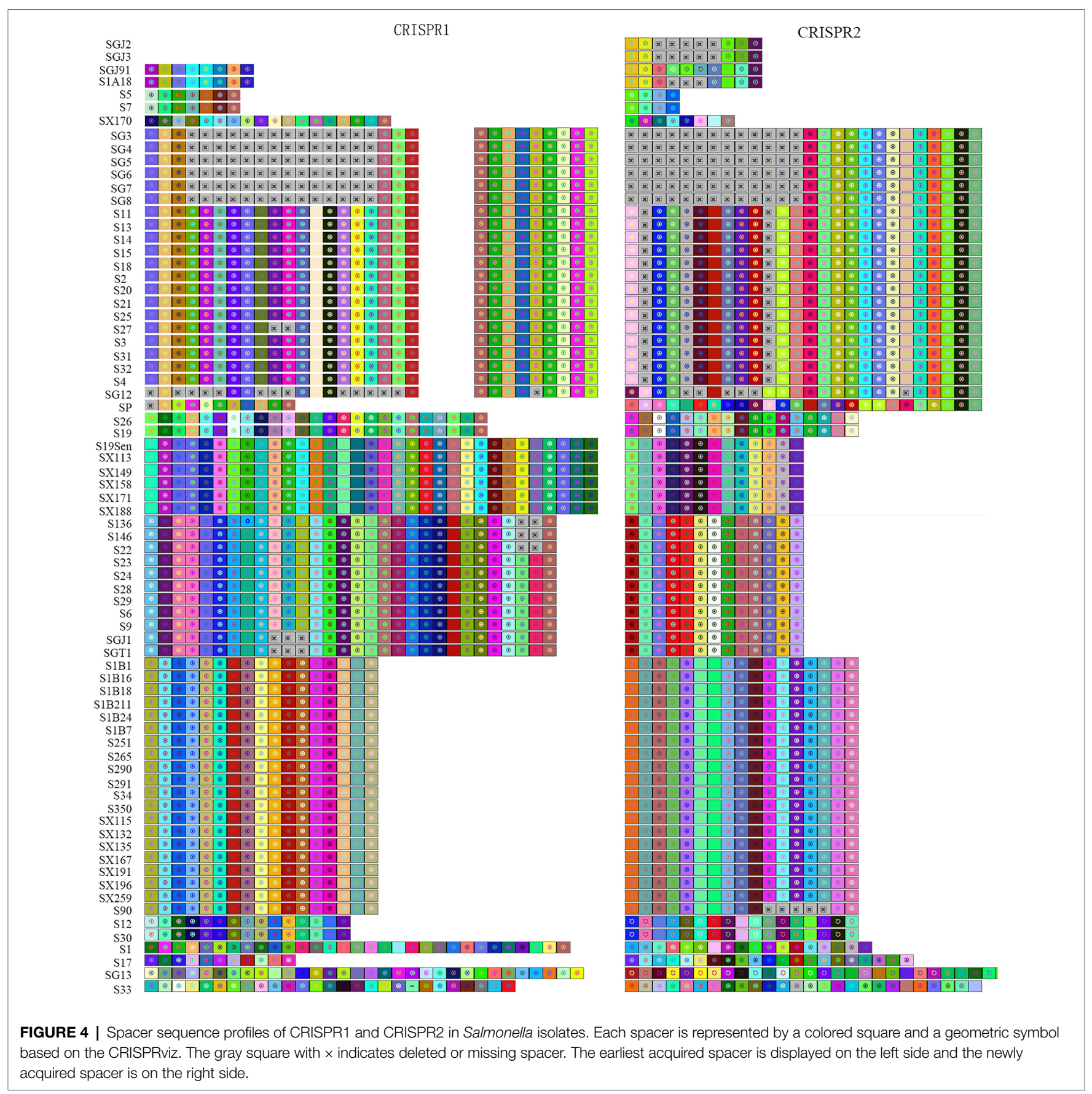

into 20 CRISPR types (Figure 4), whereas in CLSPT, 72 Salmonella strains were subtyped into 16 different CRISPR types (Figure 5A). Overall, the 72 Salmonella strains were subtyped into 17 different CRISPR types in CLTSST (Figure 5B). Among the three typing methods, the values for Simpson's index of diversity were 87.75 , 85.52 , and $86.46 \%$, respectively.

To evaluate the relationships between all detected CRISPR types and serotypes, a phylogenetic tree based on the spacer sequences of the CRISPR types was constructed. The results showed that the CRISPR types had a good correspondence with serotypes, whereas strains belonging to the same CRISPR type were usually located on the neighboring branch. For S. Typhimurium, four small divergent phylogenetic clusters were observed in CLTSST and CLSPT (Figure 5).

\section{Association Between CRISPR-Cas System and Antimicrobial Resistance}

We found that there were no differences in the numbers of spacers in CRISPR arrays after comparing CRISPR sequences between susceptible and resistant strains (Table 1 and Supplementary Table S1). We also calculated the Spearman's rank correlation coefficient between the CRISPR and antibiotics 

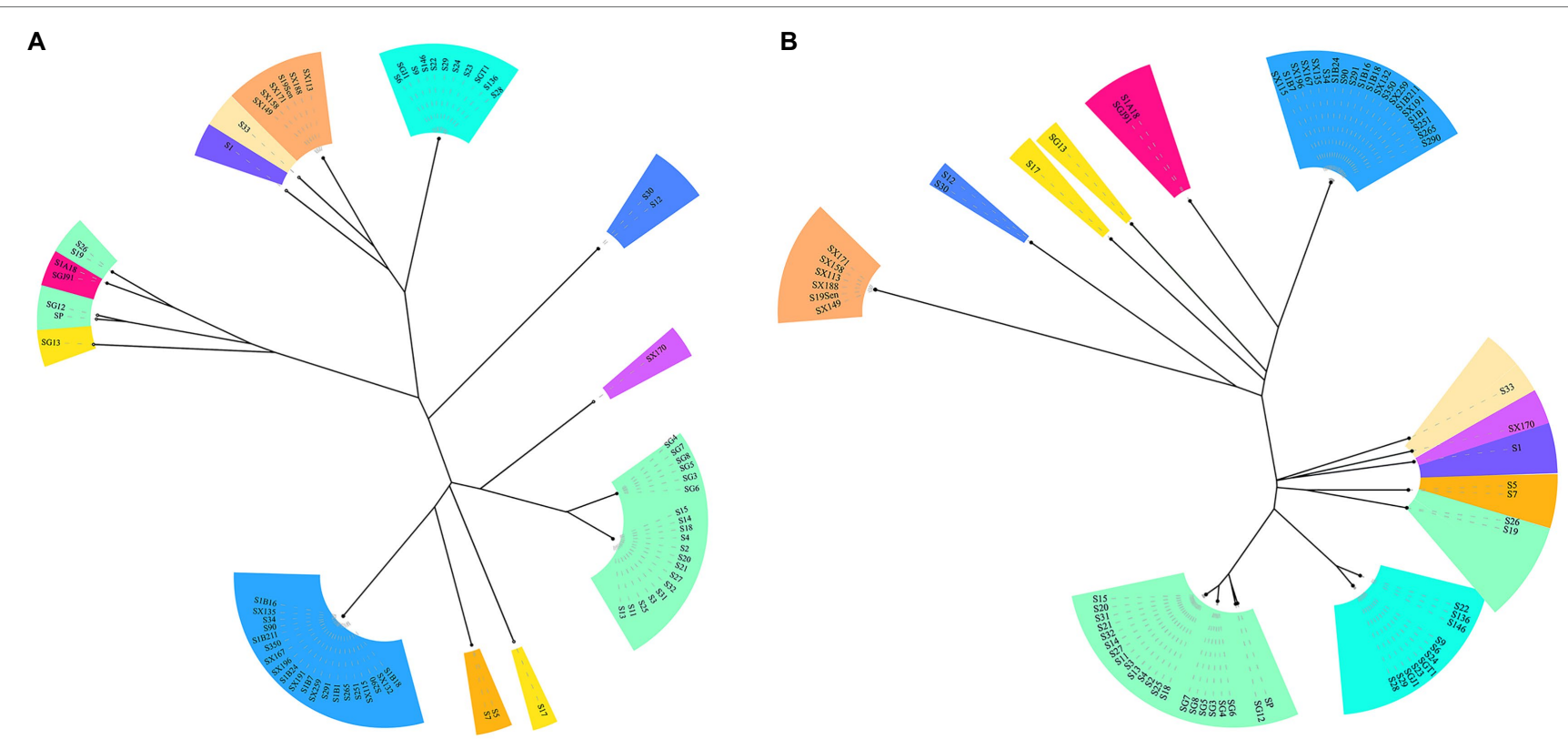

FIGURE 5 | Unweighted pair group method with arithmetic mean (UPGMA) tree of Salmonella isolates. (A) UPGMA tree based on CRISPR locus spacer pair typing (CLSPT). (B) UPGMA tree based on CRISPR locus three spacer sequence typing (CLTSST). The same color indicates the same serotype.

(Figure 6A). From the figure, the CRISPR1, CRISPR1, and CRISPR2 were correlation with aminoglycoside and trimethoprim antibiotics with a correlation coefficient of $0.23(p<0.01)$ which was indicating a modest relationship. Additionally, in total, 47 drug-resistant genes identified in the isolates were analyzed, and we found that the aac(6)-Iaa was close positive (the correlation coefficient was $0.561, p<0.01$ ) correlation with the CRISPR1 or CRISPR2 and was close negative (the correlation coefficients were -0.441 and $-0.435, p<0.01$ ) correlation with CRISPR1, CRISPR1, and CRISPR2 (Figure 6B). The $b l a_{\mathrm{TEM}-1 \mathrm{~B}}$ was close positive $(p<0.01)$ correlation with the CRISPR1 and was close negative $(p<0.01)$ correlation with CRISPR1 and CRISPR2 (Figure 6B). These results indicated that there were no close correlations between CRISPR loci and antibiotics but had close correlations between CRISPR loci and ARGs in Salmonella isolates.

\section{DISCUSSION}

Recent reports have shown that the distribution of CRISPR spacer sequences of Salmonella is related to the serotype and subtype (Fabre et al., 2012). A CLSPT method based on the newly incorporated spacer for Salmonella enterica has been constructed; this is a simplified CRISPR-based typing method with higher consistency compared with traditional serotyping (Li et al., 2014). CRISPR combined with multi-site virulence sequence typing (CRISPR-MVLST) has been established in Salmonella and has proven to be a very effective typing technique in some serotypes (Shariat et al., 2013b, 2013a). Two CRISPR spacer sequences of Salmonella were combined with two virulence genes (fimH and sseL) and MLST to type Salmonella, and the results showed that the new genotyping method could improve the resolution compared with PFGE (Liu et al., 2011). However, because the above methods ignored differences in other spacer sequences, they showed weaker typing effects. In order to make up for this shortcoming, we developed a new method to distinguish Salmonella isolates in this work. Compared with the other subtyping methods (MLST, CCT, and CLSPT), the discrimination of CLTSST $(86.46 \%)$ was weaker than that of CCT $(87.75 \%)$, but stronger than those of MLST $(85.66 \%)$ and CLSPT $(85.52 \%)$. In these CRISPR-based typing methods, the CCT needed to analyze all spacer sequences in the strain. And the CLTSST only needed to know part of the spacer sequences, which not only had good typing effect, but also greatly reduced the cost. Therefore, the CLTSST could be used as the best method for typing different serotypes. The CLSPT was a simplified version of CCT. It only needed to analyze the newly inserted spacer sequences but ignored the first three spacer sequences that best reflected the original information of the strain and the CLTSST just made up for this deficiency. However, the CLTSST still had some shortcomings. For example, when there was no CRISPR in bacteria, this method could not be used. At the same time, this method still ignored part of the spacer sequence, so this method still needed to be further improved.

In this study, the lengths of different CRISPR arrays varied greatly, with the number of spacers ranging from 4 to 34 . In some strains, spacers appeared repeatedly in multiple ways. The repetitive distribution of the spacer might be related to the sequence recombination of the repeat, and the recombination process might be accompanied by an increase and deletion of the spacer, as well as the position change of the spacer. This R-S recombination update could improve the adaptability of 


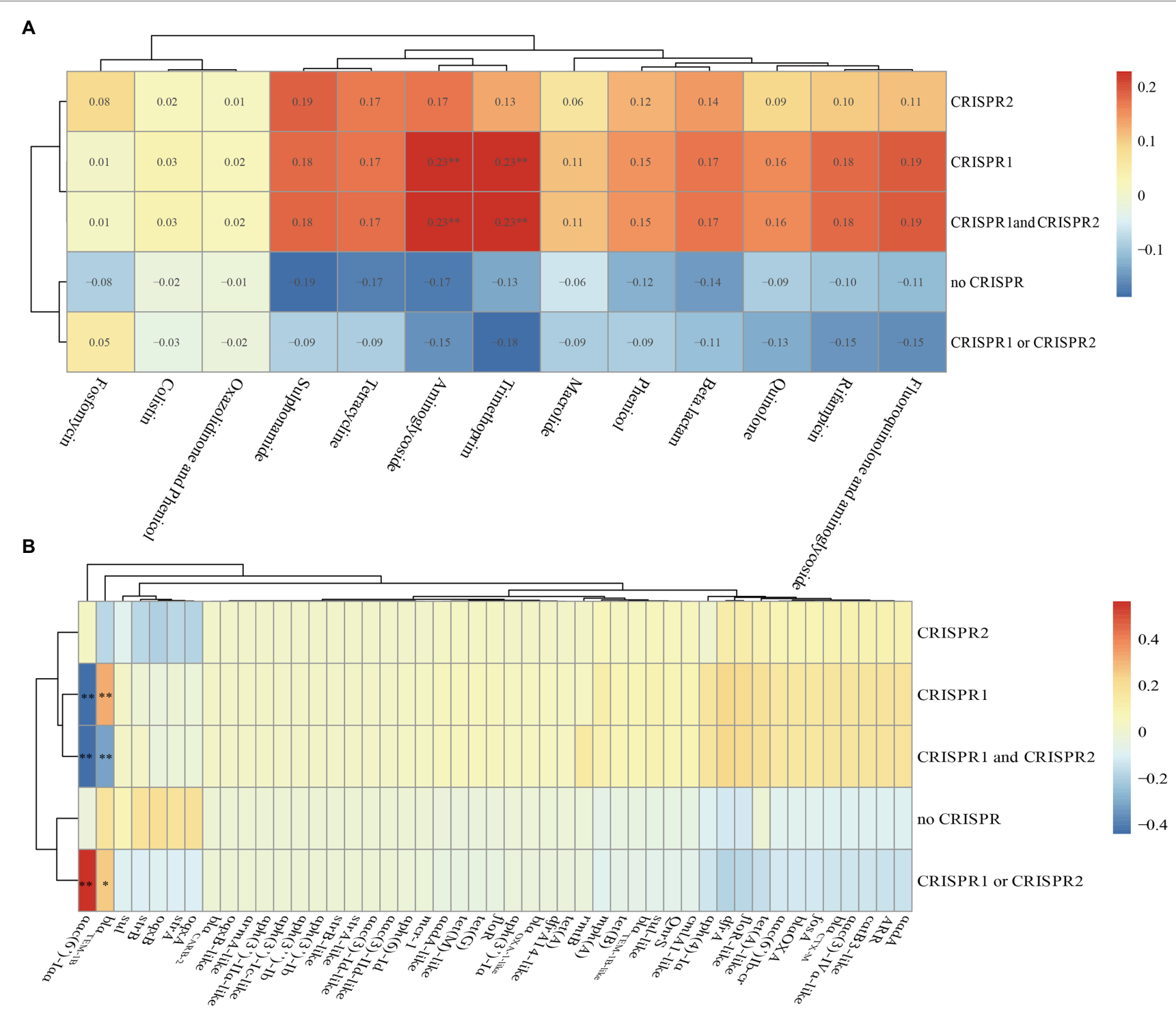

FIGURE 6 | Correlation heat map between CRISPR loci and antibiotics or antibiotic resistance genes in the total of 75 Salmonella isolates. (A) Correlation between CRISPR loci and antibiotics. (B) Correlation between CRISPR loci and antibiotic resistance genes. Correlation is expressed by Spearman correlation coefficient which is indicated by color gradient, red represents positive correlation, and blue represents negative correlation. ${ }^{* *}$ denotes $p<0.01 ;{ }^{*}$ denotes $p<0.05$. Generally, a correlation coefficient above 0.7 indicates that the relationship is very close; $0.4-0.7$ indicates that the relationship is close; and $0.2-0.4$ indicates that the relationship is normal.

the strain. The structural features and sequence functions of Salmonella CRISPR were also explored. The DR sequences were different, and part of the spacer sequences was derived from foreign genes. We speculated that under antibiotic selection pressure, the bacterial CRISPR sequence may mutate, allowing the bacteria to acquire exogenous resistant genes and survive. This suggested that the variation in CRISPR DR sequences and the diversity of spacer sequences might be related to the resistance of Salmonella. The relationships between the number of CRISPR spacers and antibiotic resistance differed according to the type of antibiotic and the species of the strain. For example, the number of CRISPR spacers was positively correlated with SXT resistance but not correlated with AMP and cefazolin resistance in uropathogenic E. coli (Dang et al., 2013). By analyzing the relationships between the CRISPR-Cas system of 263 strains of $E$. coli and drug resistance, researchers found that there were no significant differences in the distributions of cas between sensitive and resistant strains and that drugresistant plasmids could spread among CRISPR-positive E. coli (Touchon et al., 2012). However, the distribution of Enterococcus faecalis CRISPR was negatively correlated with bacterial resistance (Palmer and Gilmore, 2010). In this study, there were no close correlations between the CRISPR-Cas system and antibiotics in Salmonella isolates. But the aac(6)-Iaa was close positive correlation with the CRISPR1 or CRISPR2 and was close negative correlation with CRISPR1, CRISPR1 and CRISPR2. 
The $b l a_{\mathrm{TEM}-1 \mathrm{~B}}$ was close positive correlation with the CRISPR1 and was close negative correlation with CRISPR1 and CRISPR2. To date, no unified statement of the relationships between bacterial CRISPR and bacterial resistance to antibiotics has been proposed. This may be because of variations related to factors, such as the bacterial species, antibiotic species, host, strain isolation time, and geographic location. Therefore, further studies are needed to assess the relationships between bacterial CRISPR and antibiotic resistance.

\section{CONCLUSION}

In summary, we identified 517 unique spacers and 31 unique direct repeats in the CRISPR loci of these isolates. Further analysis of the identified spacers and repeats demonstrated specific patterns that harbored certain associations with genotype. We also established the CLTSST method and applied this method to the typing of different serotypes of Salmonella to explore the structure and function of CRISPR. Simultaneously, compared with the other three typing methods, we found that the discrimination of CLTSST was weaker than that of CCT, but stronger than those of MLST and CLSPT. In addition, we analyzed the relationships between CRISPR loci and Salmonella resistance and found that there were no close correlations between CRISPR loci and antibiotics but had close correlations between CRISPR loci and ARGs in Salmonella isolates.

\section{DATA AVAILABILITY STATEMENT}

The datasets presented in this study can be found in online repositories. The names of the repository/repositories and accession number(s) can be found at: https://www.ncbi.nlm. nih.gov/, SAMN19819839, SAMN19819840, SAMN198 19841, SAMN19819842, SAMN19819843, SAMN19819844, SAMN19819845, SAMN19819846, SAMN19819847, SAMN19 819848, SAMN19819849, SAMN19819850, SAMN19819851, SAMN19819852, SAMN19819853, SAMN19819854, SAMN198

\section{REFERENCES}

Antunes, P., Mourão, J., Campos, J., and Peixe, L. (2016). Salmonellosis: the role of poultry meat. Clin. Microbiol. Infect. 22, 110-121. doi: 10.1016/j. cmi.2015.12.004

Bankevich, A., Nurk, S., Antipov, D., Gurevich, A. A., Dvorkin, M., Kulikov, A. S., et al. (2012). SPAdes: a new genome assembly algorithm and its applications to single-cell sequencing. J. Comput. Biol. 19, 455-477. doi: 10.1089/ cmb.2012.0021

Barrangou, R., and Dudley, E. G. (2016). CRISPR-based typing and nextgeneration tracking technologies. Annu. Rev. Food Sci. Technol. 7, 395-411. doi: 10.1146/annurev-food-022814-015729

Best, E. L., Hampton, M. D., Ethelberg, S., Liebana, E., Clifton-Hadley, F. A., and Threlfall, E. J. (2009). Drug-resistant salmonella Typhimurium DT 120: use of PFGE and MLVA in a putative international outbreak investigation. Microb. Drug Resist. 15, 133-138. doi: 10.1089/mdr.2009.0911
19855, SAMN19819856, SAMN19819857, SAMN19819858, SAMN19819859, SAMN19819860, SAMN19819861, SAMN198 19862, SAMN19819863, SAMN19819864, SAMN19819865, SAMN19819866, SAMN19819867, SAMN19819868, SAMN198 19869, SAMN19820037, SAMN19820038, SAMN19820039, SAMN19820040, SAMN19820041, SAMN19819984, SAMN198 19985, SAMN19819986, SAMN19819987, SAMN19819988, SAMN19819989, SAMN19819990, SAMN19819991, SAMN198 19992, SAMN19819993, SAMN19819994, SAMN19819995, SAMN19819996, SAMN19819997, SAMN19819998, SAMN198 19999, SAMN19820000, SAMN19820001, SAMN19820002, SAMN19820003, SAMN19820004, SAMN19820005, SAMN198 20006, SAMN19820007, SAMN19820008, SAMN19820009, SAMN19820010, SAMN19812804, SAMN19812805, SAMN198 12806, SAMN19812807, SAMN19812808, SAMN19812809, SAMN19812810, SAMN19812811, SAMN19812510, SAMN198 12511, SAMN19812512, SAMN19812513.

\section{AUTHOR CONTRIBUTIONS}

HW and CuL designed the study and supervised the work. YW, YG, ChL, and BM participated, coordinated, and analyzed the data. CuL wrote the manuscript. All authors approved the final manuscript.

\section{FUNDING}

This work was supported by the National Natural Science Foundation of China (31830098 and 31772769), the China Agriculture Research System National System for Layer Production Technology (CARS-40-K14), and the Fundamental Research Funds for the Central Universities (SCU2021D006).

\section{SUPPLEMENTARY MATERIAL}

The Supplementary Material for this article can be found online at: https://www.frontiersin.org/articles/10.3389/fmicb.2021.730046/ full\#supplementary-material 
Centers for Disease Control and Prevention (2019). Outbreak of MultidrugResistant Salmonella Infections Linked to Raw Chicken Products. Available at: https://www.cdc.gov/salmonella/infantis-10-18/index.html (Accessed Febr. 21, 2019)

Chen, S., Liu, H., Liang, W., Hong, L., Zhang, B., Huang, L., et al. (2019). Insertion sequences in the CRISPR-Cas system regulate horizontal antimicrobial resistance gene transfer in Shigella strains. Int. J. Antimicrob. Agents 53, 109-115. doi: 10.1016/j.ijantimicag.2018.09.020

Clinical and Laboratory Standards Institute (2019). Lexikon der Medizinischen Laboratoriumsdiagnostik. eds. A. M. Gressner and T. Arndt (Springer Reference Medizin. Springer, Berlin, Heidelberg), 604.

Couvin, D., Bernheim, A., Toffano-Nioche, C., Touchon, M., Michalik, J., Néron, B., et al. (2018). CRISPRCasFinder, an update of CRISRFinder, includes a portable version, enhanced performance and integrates search for Cas proteins. Nucleic Acids Res. 46, W246-W251. doi: 10.1093/nar/gky425

Crooks, G. E., Hon, G., Chandonia, J.-M., and Brenner, S. E. (2004). WebLogo: a Sequence Logo Generator. Genome Res. 14, 1188-1190. doi: 10.1101/gr.849004

Dang, T. N. D., Zhang, L., Zöllner, S., Srinivasan, U., Abbas, K., Marrs, C. F., et al. (2013). Uropathogenic Escherichia coli are less likely than paired fecal E. coli to have CRISPR loci. Infect. Genet. Evol. 19, 212-218. doi: 10.1016/j. meegid.2013.07.017

Fabre, L., Zhang, J., Guigon, G., Le Hello, S., Guibert, V., Accou-Demartin, M., et al. (2012). Crispr typing and subtyping for improved laboratory surveillance of Salmonella infections. PLoS One 7:e36995. doi: 10.1371/journal. pone. 0036995

Gophna, U., Kristensen, D., Wolf, Y., Popa, O., Drevet, C., and Koonin, E. (2015). No evidence of inhibition of horizontal gene transfer by CRISPR-Cas on evolutionary timescales. ISME J. 9, 2021-2027. doi: 10.1038/ismej.2015.20

Graziani, C., Losasso, C., Luzzi, I., Ricci, A., Scavia, G., and Pasquali, P. (2017). "Salmonella," in Foodborne Diseases. eds. C. E. R. Dodd, T. Aldsworth, R. A. Stein, D. O. Cliver, H. P. Riemann.

Grissa, I., Vergnaud, G., and Pourcel, C. (2007). The CRISPRdb database and tools to display CRISPRs and to generate dictionaries of spacers and repeats. BMC Bioinf. 8:172. doi: 10.1186/1471-2105-8-172

Hunter, P. R., and Gaston, M. A. (1988). Numerical index of the discriminatory ability of typing systems: an application of Simpson's index of diversity. J. Clin. Microbiol. 26, 2465-2466. doi: 10.1128/jcm.26.11.2465-2466.1988

Jansen, R., Embden, J., Gaastra, W., and Schouls, L. (2002). Identification of genes that are associated with DNA repeats in prokaryotes. Mol. Microbiol. 43, 1565-1575. doi: 10.1046/j.1365-2958.2002.02839.x

Lange, S., Alkhnbashi, O., Rose, D., Will, S., and Backofen, R. (2013). CRISPRmap: an automated classification of repeat conservation in prokaryotic adaptive immune systems. Nucleic Acids Res. 41, 8034-8044. doi: 10.1093/nar/gkt606

Li, H., Li, P., Xie, J., Yi, S., Yang, C., Wang, J., et al. (2014). New clustered regularly interspaced short palindromic repeat locus spacer pair typing method based on the newly incorporated spacer for Salmonella enterica. J. Clin. Microbiol. 52, 2955-2962. doi: 10.1128/JCM.00696-14

Liu, F., Barrangou, R., Gerner-Smidt, P., Ribot, E. M., Knabel, S. J., and Dudley, E. G. (2011). Novel virulence gene and clustered regularly interspaced short palindromic repeat (CRISPR) multilocus sequence typing scheme for subtyping of the major serovars of Salmonella enterica subsp. enterica. Appl. Environ. Microbiol. 77, 1946-1956. doi: 10.1128/AEM.02625-10

Long, J., Xu, Y., Ou, L., Yang, H., Xi, Y., Chen, S., et al. (2020). Utilization of clustered regularly interspaced short palindromic repeats to genotype Escherichia coli Serogroup O80. Front. Microbiol. 11:1708. doi: 10.3389/ fmicb.2020.01708

Mathews, D., Disney, M., Childs, J., Schroeder, S., Zuker, M., and Turner, D. (2004). Incorporating chemical modification constraints into a dynamic programming algorithm for prediction of RNA secondary structure. Proc. Natl. Acad. Sci. U. S. A. 101, 7287-7292. doi: 10.1073/PNAS.0401799101

McWhorter, A. R., and Chousalkar, K. K. (2019). From hatch to egg grading: monitoring of Salmonella shedding in free-range egg production systems. Vet. Res. 50:58. doi: 10.1186/s13567-019-0677-4

Nethery, M., and Barrangou, R. (2019). CRISPR visualizer: rapid identification and visualization of CRISPR loci via an automated high-throughput processing pipeline. RNA Biol. 16, 577-584. doi: 10.1080/15476286.2018.1493332

Palmer, K. L., and Gilmore, M. S. (2010). Multidrug-resistant enterococci lack CRISPR-cas. MBio 1, e00227-e00310. doi: 10.1128/mBio.00227-10
Pang, J. C., Chiu, T. H., Helmuth, R., Schroeter, A., Guerra, B., and Tsen, H. Y. (2007). A pulsed field gel electrophoresis (PFGE) study that suggests a major world-wide clone of Salmonella enterica serovar Enteritidis. Int. J. Food Microbiol. 116, 305-312. doi: 10.1016/j.ijfoodmicro.2006.05.024

Salmonella (non-typhoidal) (2018). Available at: https://www.who.int/en/newsroom/fact-sheets/detail/salmonella-(non-typhoidal). (Accessed February 20, 2018).

Sanchez, S., Hofacre, C. L., Lee, M. D., Maurer, J. J., and Doyle, M. P. (2002). Animal sources of salmonellosis in humans. J. Am. Vet. Med. Assoc. 221, 492-497. doi: 10.2460/javma.2002.221.492

Shariat, N., DiMarzio, M. J., Yin, S., Dettinger, L., Sandt, C. H., Lute, J. R., et al. (2013a). The combination of CRISPR-MVLST and PFGE provides increased discriminatory power for differentiating human clinical isolates of Salmonella enterica subsp. enterica serovar Enteritidis. Food Microbiol. 34, 164-173. doi: 10.1016/j.fm.2012.11.012

Shariat, N., Kirchner, M. K., Sandt, C. H., Trees, E., Barrangou, R., and Dudley, E. G. (2013b). Subtyping of Salmonella enterica serovar Newport outbreak isolates by CRISPR-MVLST and determination of the relationship between CRISPR-MVLST and PFGE results. J. Clin. Microbiol. 51, 2328-2336. doi: 10.1128/JCM.00608-13

Shehreen, S., Chyou, T., Fineran, P., and Brown, C. (2019). Genome-wide correlation analysis suggests different roles of CRISPR-Cas systems in the acquisition of antibiotic resistance genes in diverse species. Philos. Trans. R. Soc. Lond. Ser. B Biol. Sci. 374:20180384. doi: 10.1098/ RSTB.2018.0384

Timme, R. E., Pettengill, J. B., Allard, M. W., Strain, E., Barrangou, R., Wehnes, C., et al. (2013). Phylogenetic diversity of the enteric pathogen Salmonella enterica subsp. enterica inferred from genome-wide reference-free SNP characters. Genome biol. Evolution 5, 2109-2123. doi: 10.1093/gbe/evt159

Touchon, M., Charpentier, S., Pognard, D., Picard, B., Arlet, G., Rocha, E. P. C., et al. (2012). Antibiotic resistance plasmids spread among natural isolates of Escherichia coli in spite of CRISPR elements. Microbiology 158, 2997-3004. doi: 10.1099/mic.0.060814-0

Turki, Y., Mehri, I., Fhoula, I., Hassen, A., and Ouzari, H. (2014). Comparison of five molecular subtyping methods for differentiation of Salmonella kentucky isolates in Tunisia. World J. Microbiol. Biotechnol. 30, 87-98. doi: 10.1007/ s11274-013-1414-1

Uzzau, S., Brown, D. J., Wallis, T., Rubino, S., Leori, G., Bernard, S., et al. (2000). Host adapted serotypes of Salmonella enterica. Epidemiol. Infect. 125, 229-255. doi: 10.1017/S0950268899004379

Vincent, C., Usongo, V., Berry, C., Tremblay, D., Moineau, S., Yousfi, K., et al. (2018). Comparison of advanced whole genome sequence-based methods to distinguish strains of Salmonella enterica serovar Heidelberg involved in foodborne outbreaks in Québec. Food Microbiol. 73, 99-110. doi: 10.1016/j. fm.2018.01.004

Yousfi, K., Usongo, V., Berry, C., Khan, R., Tremblay, D., Moineau, S., et al. (2020). Source tracking based on Core genome SNV and CRISPR typing of Salmonella enterica Serovar Heidelberg isolates involved in foodborne outbreaks in Québec, 2012. Front. Microbiol. 11:1317. doi: 10.3389/ fmicb.2020.01317

Conflict of Interest: The authors declare that they have no competing financial interests or personal relationships that could have appeared to influence the work reported in this paper.

Publisher's Note: All claims expressed in this article are solely those of the authors and do not necessarily represent those of their affiliated organizations, or those of the publisher, the editors and the reviewers. Any product that may be evaluated in this article, or claim that may be made by its manufacturer, is not guaranteed or endorsed by the publisher.

Copyright (c) $2021 \mathrm{Li}$, Wang, Gao, Li, Ma and Wang. This is an open-access article distributed under the terms of the Creative Commons Attribution License (CC $B Y)$. The use, distribution or reproduction in other forums is permitted, provided the original author(s) and the copyright owner(s) are credited and that the original publication in this journal is cited, in accordance with accepted academic practice. No use, distribution or reproduction is permitted which does not comply with these terms. 